\title{
La intervención social desde la extensión universitaria: un modelo en construcción
}

María Elena Kessler

mileva143@gmail.com

Marcos Exequiel Angelon

marcosangeloni@yahoo.com.ar

Sofía Clarisa Marzioni

sofimarzioni@hotmail.com

Julio César Lozeco

jlozeco@gmail.com
A 100 años de la Reforma Universitaria de 1918

Desafíos de gestión

RECEPCIÓN: 24/06/17

Dirección de Integración Extensión Investigación y Políticas Públicas de la Secretaría de Extensión de la Universidad Nacional del Litoral, Argentina.

\section{Resumen}

En el presente artículo la Dirección de Integración Extensión, Investigación y Políticas Públicas de la Secretaría de Extensión de la Universidad Nacional del Litoral propone, como marco de referencia, un modelo de intervención en el contexto de la extensión universitaria, que se nutre de las trayectorias académicas y las prácticas desarrolladas por sus integrantes. El modelo pretende aportar a la discusión teórica al señalar algunas pistas para un análisis y reflexión en torno a los modos en que se desarrollan estos procesos.

\section{Resumo}

No presente artigo a Divisão de Integração Extensão, Pesquisa e Políticas Públicas da Secretaria de Extensão da Universidad Nacional del Litoral propõe, como marco de referência, um modelo de intervenção no âmbito da extensão universitária, que se baseia na trajetória académica e nas práticas desenvolvidas por seus integrantes. O modelo pretende contribuir com a discussão teórica apontando algumas pistas para uma análise e reflexão sobre as formas como a intervenção social se desenvolve no campo da extensão universitária.

Palavras-chave

- Extensão universitária

- Modelo de intervenção

- Políticas públicas

\section{Para citación de este artículo}

Kessler, M. E., Angeloni, M. E., Marzioni, S. C. y Lozeco, J. C. (2017). La intervención social desde la extensión universitaria: un modelo en construcción. Revista $+E$ versión impresa, 7(7), 88-99. Santa Fe, Argentina: Ediciones UNL. 
"En el contexto actual, la intervención en lo social se nos presenta como un espacio de libertad, ya que se construye en pequeños hiatos, intersticios, lugares, donde es posible reconstruir historicidad, entender a ese otro no como un sujeto a moldear sino como un portador de historia social, de cultura, de relaciones interpersonales. Tal vez la intervención en lo social no implique agregar ni quitar nada sino solamente 'hacer ver' aquello que el contexto, el escenario, el clima de época impiden visualizar." (Carballeda, 2002:32)

\section{Introducción}

El trabajo se organiza en cuatro partes. En primer lugar, presenta la Dirección, su misión y sus funciones. En segundo lugar, aborda la cuestión de la articulación entre la investigación y la extensión, por un lado, y la extensión y las políticas públicas, por otro. Luego, se desarrollan los pilares en los que se sustenta el modelo de intervención y, finalmente, se formulan unas reflexiones, a modo de interrogantes, en torno a los procesos que la intervención social promueve en el escenario de la extensión.

\section{Presentación de la Dirección de Integración Extensión, Investigación y Políticas Públicas}

El presente artículo surge del trabajo realizado en la Dirección de Integración Extensión, Investigación y Políticas Públicas de la Secretaría de Extensión de la Universidad Nacional del Litoral (UNL). La misma fue creada en el año 2015 con la misión de aportar a la generación de mecanismos y estrategias que promuevan la articulación de las funciones de extensión con la investigación y las políticas públicas, de manera de favorecer procesos de apropiación social del conocimiento, perspectivas críticas respecto de las políticas públicas, como también aprendizajes compartidos con los actores sociales involucrados en los procesos que se construyan colectivamente. Para ello cuenta con un conjunto de dispositivos, particularmente: el Sistema Integrado de Programas y Proyectos (SIPPE), ${ }^{1}$ intervenciones territoriales y su vinculación con el Estado en sus distintas jurisdicciones. Identifica además aquellos proyectos de extensión que por la trayectoria que acreditan pueden canalizarse hacia proyectos de investigación, especialmente en el marco del programa Curso de Acción para la Investigación y Desarrollo (CAl+D) Orientado a Problemas Sociales y Socioproductivos ${ }^{2}$ (u otros programas de investigación).
Las funciones que la Dirección desarrolla pueden sintetizarse en: - Asistir y asesorar al secretario de Extensión en materia de políticas y acciones vinculadas a la integración de la extensión con la investigación, así como la participación y aportes a las políticas públicas, tanto municipales, provinciales como nacionales.

- Contribuir al Programa CAI+D Orientado a Problemas

Sociales y Socioproductivos u otros programas de investigación similares, tanto en la identificación de las agendas socialmente consensuadas para la investigación como en la participación de sectores sociales e institucionales en los procesos de construcción de conocimientos.

- Analizar y desarrollar acciones en conjunto con las Secretarías de Ciencia y Técnica y de Vinculación Tecnológica de la UNL en materia de apropiación social del conocimiento y de innovación social. - Diseñar estrategias de vinculación con las políticas públicas (municipal, provincial y/o nacional) a través de acciones, proyectos y programas de extensión.

- Promover, a través de la Cátedra Abierta de Extensión y Políticas Públicas, instancias de formación, debate y reflexión con relación al vínculo universidad-Estado-sociedad para la construcción, desarrollo y evaluación de políticas públicas.

\section{De la extensión, la investigación y las políticas públicas}

A continuación, para dar cuenta de las lógicas de la extensión y la investigación que la UNL desarrolla, se hará mención a los distintos dispositivos que ha ido diseñando. En su articulación con la sociedad y el Estado, la UNL ha implementado múltiples instrumentos de gestión. En cuanto a la extensión, entre los más destacados se encuentra el Sistema Integrado de Programas y Proyectos de Extensión, en marcha desde el año 1995 (Resolución del Consejo Superior $\left.N^{\circ} 143 / 95\right)$. El mismo cuenta con la participación de estudiantes, docentes y graduados, voluntarios y representantes de la comunidad. Está conformado por programas institucionales y diversas modalidades de proyectos: Proyectos de Extensión de Interés Social (PEIS), Acciones de Extensión al Territorio (AET) y Proyectos de Extensión de Interés Institucional (PEII), los cuales abordan problemáticas diversas y establecen para ello nexos directos con organismos públicos y con organizaciones de la sociedad civil.

El Sistema promueve la incorporación curricular de la extensión; la apropiación social de los conocimientos; la identificación de nuevas líneas de investigación; la integración de la extensión con
1) EI SIPPE se propone como objetivo principal la consolidación de los procesos de gestión y de las capacidades institucionales en extensión. Los
Programas y Proyectos de Extensión son los dispositivos de gestión que posibilitan la articulación y vinculación de los equipos académicos con los grupos y organizaciones sociales. 2) Por intermedio del programa $\mathrm{CAl}+\mathrm{D}$ Orientado a Problemas Sociales y Socioproductivos, la UNL profundiza el conocimiento y aporta soluciones sobre problemas que la comunidad y el desarrollo sostenible de la región demandan. 
la docencia y la investigación; la interdisciplina y la integración de docentes, estudiantes, graduados y referentes comunitarios. Asimismo, en su contexto se analizan y desarrollan convocatorias de proyectos realizadas por organismos externos a la Universidad, tales como las de la Secretaría de Políticas Universitarias (SPU) y el Voluntariado Nacional (ambos pertenecientes al Ministerio de Educación y Deportes de la Nación), y también las de otros organismos públicos.

El objetivo principal de la extensión universitaria es la promoción del desarrollo local y regional y la mejora en la calidad de vida de los ciudadanos a partir de diversas líneas de acción vinculadas a temáticas y/o problemáticas del medio socioproductivo y el fomento de espacios de articulación con las prácticas en docencia y en investigación. En este sentido, los distintos tipos de proyectos de extensión mencionados —más allá de sus diferentes modalidades- presentan las características de proyectos de desarrollo social, ya que cada uno de ellos está relacionado con un conjunto de problemáticas identificadas, vinculadas a un sector social definido y localizado en un determinado territorio. Esta dinámica de trabajo resignifica el sentido de pertenencia de la Universidad y su función social:

"Se profundizan los objetivos planteados en el vínculo de la universidad con la sociedad, en el que no sólo se intenta contribuir al bienestar y la calidad de vida de la población sino que además se plantea esta relación en base a una profunda necesidad académica que enriquece las líneas de investigación, las propuestas curriculares de las carreras y la generación de nuevas líneas de extensión". (Menéndez, 2012:9)

En lo que respecta a la investigación, dentro de los distintos dispositivos existentes se encuentra el Programa Cursos de Acción de Investigación y Desarrollo ( $\mathrm{CAl}+\mathrm{D})$, de perfil científico tecnológico, como aquellos destinados a Problemas Sociales y Productivos (CAl+D Orientado). Estos últimos tienen como objetivo la generación de conocimientos en el contexto de aplicación e incorporan la participación de los actores territoriales en las etapas de formulación y ejecución de los mismos.

De esta manera, el Programa tiende a vincular los espacios de producción de conocimientos con los de su uso y apropiación social, a incentivar la investigación científica orientada a la atención de problemas sociales y productivos de la región, promover la investigación conducente a resultados socialmente relevantes, y alentar la interdisciplinariedad en los procesos.

Esta perspectiva abreva en los cambios producidos a partir de la década de 1970 en cuanto a la noción de conocimiento como construcción social. Desde aquel entonces, y como respuesta a las lecturas funcionalistas, se fue dando un significativo giro conceptual y también metodológico al hacer énfasis en las influencias de los procesos sociales sobre la producción de conocimiento científico.
Los trabajos de Bloor (1976) y Barnes y Edge (1982) han criticado una visión de la producción científica como actividad autorregulada e independiente frente a factores externos. El debate en cuanto a la veracidad del conocimiento científico pierde centralidad en la sociología del conocimiento para dar paso al estudio de las relaciones sociales que dan lugar a un tipo específico de saber, que al mismo tiempo no es más legítimo o verdadero que otras formas de conocimiento. La ciencia es, en este sentido, un producto histórico y, por lo tanto, no es posible comprender su dinámica si se prescinde del contexto social que signa estos modos de saber. Un segundo giro en la perspectiva constructivista ha estado ligado a esta última dimensión en tanto proceso histórico. Tal como ilustra el trabajo de Collins (1983), donde el foco de análisis está puesto en las controversias y tácticas a través de las cuales los argumentos y proposiciones son negociados hacia el interior de una comunidad científica, donde la ciencia no sería ya una actividad lineal, acumulativa o exenta de conflicto. Historizar el conocimiento científico ha implicado en el mismo pasaje tomarlo como proceso antes que como producto acabado. Estudios recientes (Callon y Latour, 1986) abordan en la perspectiva procesual las estrategias desplegadas por el actor al momento de "hacer ciencia", tomando como premisa que el científico es también, de manera indisociable, un actor social, y que las formas de conocer se hallan ineludiblemente atravesadas por la estructura social y la cosmovisión de una sociedad en una época.

Actualmente, coexisten perspectivas constructivistas con otras propuestas más vinculadas a la caracterización y descripción de las formas que asume el conocimiento en la sociedad contemporánea. Por otro lado, la extensión universitaria, en su dimensión territorial e institucional, está íntimamente vinculada a las políticas públicas, entendidas estas como aquellas intervenciones del Estado expresadas en una serie de decisiones intencionalmente coherentes, tomadas por una autoridad pública competente, que consideran un análisis técnico-racional a fin de resolver un problema definido públicamente como colectivo y que sigue un procedimiento institucional formal, todo lo cual se da dentro de un intenso proceso político de confrontación y articulación de intereses (Subirats et al., 2008).

El análisis de las políticas públicas no solo implica reconocer las estrategias desarrolladas por el Estado para afrontar la cuestión social, sino que también lleva a indagar en torno a las normas emanadas de los actores públicos y a las prácticas que despliegan, que incluyen las formas concretas de intervención, la reglamentación, los modos y mecanismos de gestión, la provisión de prestaciones, el alcance, los discursos y campañas de comunicación, sus finalidades y valoraciones.

En la Dirección, el estudio de las políticas públicas se aborda desde dos perspectivas: en primer lugar, como intervención estatal sobre lo social, las políticas sociales como generadoras de asociaciones 
la territorialidad adquiere relevancia no solo desde el punto de vista topográfico, sino como un espacio de significantes que remite a la idea de pertenencia, como construcción de identidades

y disputas entre distintos actores que interactúan en el territorio. En segundo lugar, las políticas públicas como formulaciones, discursos, argumentos que se despliegan para justificar cierta estrategia de intervención sobre lo social. Las políticas públicas son acciones que el Estado realiza en un contexto histórico en particular, y sin duda remiten al modelo de acumulación vigente como también al desarrollo de las políticas a nivel global. Reflejan los vínculos existentes entre los habitantes de un territorio y las agencias gubernamentales y los mecanismos de distribución del poder. A su vez, constituyen espacios de interacción social donde se definen intercambios entre sujetos que participan en un campo compuesto por relaciones históricas objetivas, entre posiciones que desnudan formas de capital simbólico y material.

\section{La intervención social pensada desde la extensión: alcances y sentidos}

La búsqueda de una mirada crítica de la intervención social es la que ha llevado al equipo de la Dirección a "ensayar" una metodología de intervención que ponga de manifiesto la complejidad que encierran los espacios multiactorales entre la Universidad, los actores sociales (miembros de organizaciones de la sociedad civil, referentes de las redes territoriales, experiencias del campo popular, entre otros) y el Estado (a través de las políticas públicas y las agencias gubernamentales) y la necesidad de definir estrategias de desarrollo territorial que inciden favorablemente en los procesos de vulnerabilidad y exclusión social.

La intervención social se muestra como construcción de lazos sociales y como búsqueda de significados en un escenario, como ese lugar en el que se desarrolla la trama social, donde el sujeto y su entorno prueban la vulnerabilidad o eficiencia de sus vínculos (Carballeda, 2005). Remite a una determinada posición en el campo social, desde la perspectiva planteada por Bourdieu y Wacquant (1995), como espacio de conflictos y competencias, estructura de probabilidades, recompensas, ganancias o sanciones, que siempre refleja cierto grado de indeterminación. En este entramado, la territorialidad adquiere relevancia no solo desde el punto de vista topográfico, sino "del lugar" como un espacio de significantes que remite a la idea de pertenencia, como construcción de identidades. La identidad como proceso de continuidades y rupturas; continuidades con referencia a prácticas, rutinas; rupturas producto de profundas transformaciones, que ponen en crisis lugares y saberes, que llevan a plantear y a construir marcos conceptuales desde donde comprender las nuevas complejidades en las que se desarrolla la vida de los sujetos, con relación a sí mismos, a sus pares y respecto del lugar que habitan.

Entendemos a la intervención social como acción que se realiza de manera formal u organizada, que responde a necesidades sociales e intenta incidir significativamente en la interacción de las personas y aspira a una legitimación pública o social. Concebida como esquema de acción, obedece a necesidades sentidas y se considera como la oportunidad para el reconocimiento del "otro" como sujeto portador de historicidad, de cultura, de lazos, en un horizonte de sentido que pasa por la integración social.

Los pilares en los que se sustenta el modelo de intervención - concebido a modo de marco referencial- incorporan claves conceptuales desde donde entender la extensión, como también un aspecto instrumental-operativo que incluye mecanismos para la participación y convalidación social de los procesos que tienen lugar en el espacio de la extensión. Los mismos dan cuenta de: 1) las dimensiones de la extensión universitaria; 2) el contexto de la intervención (en el que se proponen cuatro ejes de análisis que contemplan: las políticas sociales, el mundo del trabajo y sus mutaciones, la agenda socioambiental y los usos de las nuevas tecnologías en la cuestión ciudadana); 3) los aportes de la teoría social; 4) una perspectiva epistemológica; 5) una perspectiva ética, y 6) el aporte instrumental-operativo. 


\section{Las dimensiones de la extensión universitaria}

Desde la UNL, la extensión universitaria es considerada una de las funciones sustantivas, junto con la investigación y la docencia. Dentro de esta función, se reconocen, siguiendo los planteos de Gustavo Menéndez (2011), cinco dimensiones que le son propias: - Dimensión académica-institucional. Considera el aporte de la extensión a la construcción y apropiación social del conocimiento. Genera dispositivos de gestión que apuestan al fortalecimiento de las instituciones democráticas y de las políticas públicas.

- Dimensión social. Reconoce a la Universidad como institución social que construye y se construye en las dinámicas sociales, culturales y productivas, en un tiempo y espacio determinado, definiendo a las prácticas de extensión en el horizonte de la inclusión y la cohesión social.

- Dimensión comunicacional. Comunicación dialógica en procesos de interacción y construcción mutua con grupos, instituciones y poblaciones. Pensada como antítesis de la imposición y dominio de un saber o saberes. Una perspectiva comunicacional que sume esfuerzos en la reflexión crítica del conocimiento.

- Dimensión pedagógica. Se privilegia la educación como dispositivo que promueve prácticas de producción de conocimiento desde una perspectiva interdisciplinaria.

- Dimensión política. En tanto toda acción tiene por objeto transformar una situación determinada, interpela necesariamente al Estado y a las políticas públicas.

Estas dimensiones promueven una mirada integral que involucra a toda la comunidad universitaria, apartándose de la mirada estanca y sectorial para avanzar hacia procesos de interacción, que ponen en diálogo los espacios universitarios con la diversidad de actores y la complejidad del territorio.

\section{Contexto de la intervención}

El contexto de la intervención se refiere a la arena donde se inscriben las prácticas de extensión. Para su análisis consideramos cuatro ejes que pretenden hacer una aproximación a la cuestión social contemporánea:

- Las políticas sociales: ¿estrategia de inclusión?

- El mundo del trabajo: sus mutaciones.

- La agenda socioambiental: una problemática pendiente en la esfera pública.

- Usos de las nuevas tecnologías en la cuestión ciudadana.

Las políticas sociales: ¿estrategia de inclusión?

Una de las principales dificultades que las sociedades latinoamericanas han enfrentado en el pasado reciente -y continúan haciéndolo hoy — ha sido la desigualdad social producto de los altos niveles de pobreza e indigencia y del gran tamaño de los sectores informales (Mazzuca, 2012). Así, la cuestión social expresa lo que puede denominarse "la tensión fundante", esto es, la contradicción entre la igualdad-autonomía jurídico-política y la desigualdadheteronomía socioeconómica. En este escenario, las políticas sociales forman parte del conjunto de arreglos, siempre transitorios, referidos a la reproducción ampliada de la vida, que permiten mantener cierto orden social (Grassi, 2000).

En este sentido, se entiende aquí a las políticas sociales como aquellas intervenciones sociales

"que regulan las formas en que la población se reproduce y socializa (sobrevive físicamente y se inserta en el mundo del trabajo y en el espacio sociocultural del Estado-nación) y que protegen a la población de situaciones que ponen en riesgo esos procesos". (Andrenacci y Soldano; 2006:32)

Mediante la distribución de recursos estatales, las políticas sociales definen las responsabilidades y derechos de los ciudadanos e inciden en la estructura de distribución de los recursos societales y en la construcción de un determinado "perfil de sociedad" (Rofman; 2016). En este ejercicio, pueden tanto corregir o compensar las desigualdades que produce el mercado como generarlas y modularlas, naturalizándolas e institucionalizándolas.

En Argentina, los problemas sociales alcanzaron un punto dramático luego del colapso de la estructura macroeconómica neoliberal durante los años 2001-2003: los niveles de pobreza e indigencia se incrementaron notoriamente (54\% y $25 \%$ en 2003, respectivamente) y el desempleo llegó a su récord histórico (18,3 \% en 2001 y $21,5 \%$ en 2002) (Alonso y Di Costa; 2011). Ante esta situación, el kirchnerismo se presentó como un proyecto político destinado a superar la crisis a través de la sustitución del modelo económico excluyente por uno basado en la intervención del Estado y en políticas públicas universales como estrategia de inclusión social (Zelanik, 2012).

El período iniciado en el año 2003 marcó una ruptura con el pasado. Como es sabido, durante el período neoliberal, el modelo de protección social argentino se caracterizó por la privatización de los sistemas de salud, educación y jubilaciones, y por restringir su alcance a las personas formalmente integradas al mercado de trabajo y a las "poblaciones-problema", para las cuales se diseñaban acciones focalizadas de reducción de la pobreza. Se recuperó progresivamente el control estatal sobre la gestión de los sistemas de salud, educación y jubilaciones, y la seguridad social ha tendido a universalizarse mediante la combinación de herramientas contributivas y no contributivas.

Aun cuando el Estado recuperó su función regulatoria y algunas de sus políticas mostraron una vocación universalista (y la dinámica del gasto social acompañó estas reformas), no se cuenta con información empírica relevante, para sostener que se configuró un nuevo modelo de protección social que colocó en primer plano al ciudadano. Las reformas emprendidas han sido insuficientes para revertir las consecuencias de la retracción estatal de los 
años previos, al tiempo que han conformado un conjunto de intervenciones sectoriales que tienen limitaciones para afrontar los problemas sociales que afectan a vastos sectores de la población, incluyendo las enormes diferencias entre las jurisdicciones provinciales (Repetto, 2008).

En la perspectiva de Isuani (2015), no es correcto hablar de un nuevo modelo de protección social, más bien se fue delineando una configuración fragmentaria e híbrida, en tanto la recuperación de la centralidad del Estado y las transformaciones de la política social coexistieron con concepciones sobre la protección social, modalidades de intervención y formas de organización administrativo-institucional típicas del período neoliberal. Como resultado, pese a que presentaron rasgos estructurales, las transformaciones no fueron necesariamente "integrales" y dejaron sin resolver muchos de los antiguos desafíos de la política social argentina (Danani y Hintze, 2013).

Desde diciembre de 2015, Argentina presenta otra configuración política debido a la asunción de un gobierno de nuevo cuño partidario, que enfrenta problemas económicos (alto nivel de inflación), sociales (alto nivel de pobreza y trabajo informal), políticos (debilitamiento de las instituciones democráticas) y éticos, ligados a niveles de corrupción significativos. Sus intervenciones han desencadenado consecuencias adversas para los sectores más desfavorecidos. Según el INDEC, hacia fines de 2016 un 30,3 \% de la población argentina se encontraba en situación de pobreza y un $6,1 \%$ en situación de indigencia. En cuanto al empleo, los resultados de la Encuesta Permanente de Hogares (EPH) pusieron de relieve que un 9,2 \% de la población económica activa estaba desempleada durante el primer trimestre de 2017. De acuerdo con los datos del Sistema Integrado Previsional Argentino (SIPA), entre noviembre de 2015 y enero de 2017 , unas 48.900 personas quedaron sin trabajo en el sector privado. A la vez, se profundizó la desigualdad social: entre el segundo trimestre de 2015 y el de 2016, la brecha de ingresos entre los hogares más pobres y los más ricos pasó de 12,3 veces a 13,8 (CELS, 2017).

La definitiva consolidación de un nuevo y más incluyente modelo de protección social en Argentina, que articule las que han sido reformas valiosas con nuevas estrategias y afronte integralmente las desigualdades sociales y territoriales, demanda atender a los desafíos aún pendientes en la gestión de lo social. Entre los más urgentes se cuentan: 1) aumentar la capacidad, competencia y liderazgo político en lo más alto del Poder Ejecutivo para definir el rumbo estratégico de la política social, y lograr la articulación y sinergia entre las diversas áreas del Estado nacional para actuar integralmente frente a los problemas sociales; 2) revisar la división de tareas entre los tres niveles gubernamentales, para lo cual no solo se deben analizar las ventajas comparativas sino también definir las eventuales armonizaciones entre qué hace cada gobierno en términos sociales y qué correspondencia tiene con sus responsabilidades en materia de recaudación tributaria y gasto social; 3) mejorar la articulación entre los componentes públicos de seguridad social y privados, así como la regulación de esos últimos, para garantizar la calidad homogénea, por ejemplo de la salud y la educación, independientemente del poder adquisitivo o el tipo de acceso de cada persona; 4) repensar el financiamiento de la política social en un contexto en donde el empleo y la seguridad social centrados exclusivamente en sistemas contributivos han perdido capacidad integradora y de protección (Repetto, 2014).

Las mutaciones en el mundo del trabajo

El concepto "trabajo" es polivalente y congrega tres grandes dimensiones: a) como factor de producción, b) como esencia del hombre, y c) como sistema de distribución de ingresos.

La matriz del Estado de Bienestar integra las dimensiones aludidas en una única concepción, la del "trabajo asalariado". Desde finales del siglo XX, el trabajo asalariado formal fue perdiendo su rol de principal satisfactor de integración social y dio lugar al surgimiento de una diversidad de formas de trabajo e inserción laboral a través de las cuales las personas satisfacen su bienestar (intra e intergeneracional) que no ha sido acompañado por instituciones, normativas y regulaciones que lo contemplen (Ackerman, 2007). Esta transformación se profundizó con el advenimiento del neoliberalismo y la promoción del mercado como mecanismo regulador eficiente, perdió centralidad la relación salarial y habilitó el surgimiento de "nuevas formas de empleo" asociadas a la eficiencia, la flexibilización y descentralización de las relaciones laborales, entre otras; todo lo cual condujo a la construcción de un mundo del trabajo donde priman la fragmentación, el cambio tecnológico y la precarización y, con ello, la pérdida de la estabilidad y seguridad en el empleo y el poder de negociación de los sindicatos.

Esta consecuencia, lejos de eliminar la discusión en torno al trabajo, lo ha puesto en las principales agendas políticas y ha gestado nuevas perspectivas: desde miradas más optimistas (que aseguran que la flexibilidad interna implica una modalidad laboral más creativa e integrada, donde el trabajador se sienta "liberado") e innovadoras (basadas en el peso de la tecnología como nuevo articulador, donde el trabajo presentará una mejora permanente en sus competencias), hasta posicionamientos que cuestionan la centralidad del trabajo en la nueva reestructuración social ("trabajar menos para vivir más", el cambio de la sociedad salarial a la sociedad de las comunicaciones y de la intersubjetividad). Lo cierto es que la sociedad salarial se ha transformado, por diversas razones (de mercado, tecnológicas, de reestructuración social), y eso obliga a señalar algunos elementos característicos: - Una marcada disminución del trabajo industrial dentro del trabajo asalariado.

- Un avance del empleo informal, con predominio de empleos 
precarios, inseguros y con bajos salarios (contratos de prestación de servicios, pasantías, becas, trabajo a tiempo parcial).

- Una reestructuración productiva hacia empresas modernizadas pero que aún no logra vencer la preponderancia de empresas tradicionales.

- El resurgimiento de nuevos movimientos de lucha por el empleo no ligados a la reivindicación sindical sino a la dignidad del trabajo como satisfactor de necesidades.

- La reaparición de acciones de trabajo colectivo vinculado tanto a un nuevo cooperativismo como a nuevas prácticas asociativas.

En este contexto, la última década en Argentina estuvo fuertemente relacionada con la generación de empleo desde las políticas públicas focalizadas en la implementación de programas de asistencia e inclusión, recuperando la centralidad del Estado como pretendido promotor del crecimiento y el desarrollo. Sin embargo, estas políticas no han torcido el componente estructural del sistema de mercado: persisten grandes diferencias entre quienes tienen una relación de dependencia (y seguridad social) y quienes aún son parte de las lógicas de precarización y eficiencia productiva. El empleo público y la informalidad siguen siendo dos elementos centrales en la composición del empleo en nuestro país. Su permanencia en el tiempo constituye una asignatura pendiente y un desafío, pues atenta contra la calidad y la dignidad del trabajo (Neffa et al., 2010).

\section{La agenda socioambiental: una problemática pendiente en la esfera pública ${ }^{3}$}

Actualmente, en nuestra región se ha generalizado un modelo extractivo-exportador, que amplía todavía más las brechas existentes entre los países del norte y del sur, basado en la extracción de recursos naturales no renovables, la extensión del monocultivo, la contaminación y la pérdida de biodiversidad. Ejemplos de esta nueva división territorial y global del trabajo dentro del capitalismo actual son: el modelo de agronegocios, la megaminería a cielo abierto, la construcción de grandes represas, los proyectos previstos por la Iniciativa para la Integración de la Infraestructura Regional Suramericana (IIRSA) ${ }^{4}$ y los agrocombustibles.

Esta etapa, según Harvey (2004), puede ser definida como de "acumulación por desposesión" y coloca en el centro de la disputa la cuestión acerca del territorio y el medioambiente. No es casualidad que en este escenario de reprimarización de la economía, caracterizado por la presencia desmesurada de grandes empresas transnacionales, se hayan potenciado las luchas ancestrales por la tierra de los movimientos de indígenas y campesinos y, al mismo tiempo, hayan surgido nuevas formas de movilización y participación ciudadana, centradas en la defensa de los recursos naturales (señalados como "bienes comunes"), la biodiversidad y el medioambiente. Esto coloca la temática de lo que se entiende como "desarrollo sustentable" en la agenda política.

La afirmación de que existen regiones marcadas históricamente por la pobreza y la vulnerabilidad social, con una densidad poblacional baja, con grandes extensiones de territorios "improductivos" y/o "vacíos", facilita la instalación de un discurso productivista y excluyente, al tiempo que constituye el inicio de otros lenguajes de valoración del territorio por parte de las comunidades afectadas. Estos lenguajes de valoración distintos fueron apropiados por diferentes comunidades rurales vulneradas y también por organizaciones urbanas, fundamentalmente en Perú, Bolivia, Ecuador y Argentina. Dentro de estos movimientos socioambientales actuales, ha cobrado notoriedad el concepto de "Buen vivir" planteado como una oportunidad para construir otra sociedad y un estilo de desarrollo alterno al progreso material, sustentado en la convivencia del ser humano en diversidad y armonía con la naturaleza a partir del reconocimiento de los valores culturales existentes en cada país. Estos grupos recomiendan dejar de ver a los recursos naturales como una condición para el crecimiento económico o como un simple objeto de las políticas de desarrollo y apuntan a crear relaciones dinámicas y constructivas entre el mercado, la sociedad y el Estado. ${ }^{5}$

Si bien estas nuevas concepciones, como la del "Buen vivir", no están exentas de tensiones, la necesidad de pensar y poner a prueba nuevos modelos de desarrollo resulta ineludible. La problemática socioambiental adquiere relevancia ya que constituye un desafío permanente para el Estado, la academia y la sociedad civil, en la construcción de un mundo que apueste a un "desarrollo con rostro humano", esto es, que garantice la realización de las necesidades sociales en términos tanto materiales como subjetivos.

Los problemas socioambientales relevantes para Santa Fe Teniendo en cuenta diferentes diagnósticos realizados por diversas fuentes, provenientes tanto del mundo académico como de organizaciones sociales que intervienen en problemáticas
3) Colaboró en la elaboración de la temática abordada la bioquímica Graciela Ethel Bordón.

4) La Iniciativa para la Integración de la Infraestructura Regional Surameri- cana (IIRSA) es el foro de diálogo del que participan las 12 repúblicas de Sudamérica (actual Unión de Naciones Suramericanas) y tiene como objetivos la planificación y desarrollo de proyectos para el mejoramiento de la infraestructura regional de transporte, energía y telecomunicaciones. Para más información, ver: http://www. iirsa.org/
5) Para profundizar sobre este tema ver, por ejemplo, Salvador Schavelzon (2015). 
socioambientales, es posible encontrar coincidencias, las que a continuación se identifican: ${ }^{6}$

Santa Fe es una de las provincias más vulnerables a los efectos del cambio climático; importantes masas de agua y grandes sequías alternan sobre su territorio en forma creciente y amenazan su sustentabilidad económica y social. Todos los indicadores de pérdida de biodiversidad muestran que la provincia tiene una de las mayores tasas del país, con graves impactos en los ecosistemas pampeanos, chaqueños y fluviales, y pérdida difícil de revertir en los ecosistemas del espinal.

Estas dos realidades son consecuencia en un $50 \%$ de cuestiones globales, y en el otro $50 \%$ de responsabilidades locales representadas por un equivocado ordenamiento territorial y la matriz socioproductiva que fomentó indiscriminadamente la soja sobre la ganadería y los cultivos sustentables, la disecación y eliminación de humedales sobre su conservación, y la deforestación en la cuña chaqueña y las cuencas hídricas sobre la preservación del bosque nativo. Santa $\mathrm{Fe}$, a través de sus universidades, entes federales y el sector no gubernamental, tiene una "masa crítica" de científicos y técnicos ambientales que está por encima de la media nacional y, hasta ahora, casi no fue convocada por las políticas públicas provinciales. Por otra parte, es una de las provincias más ricas de la Argentina pero tiene una de las tasas más bajas de inversión ambiental sobre su PBI.

Otro aspecto preocupante remite al proceso de urbanización desmedido en las últimas décadas. Los nuevos procesos de desconcentración y descentralización asociados a la contraurbanización detectados en el área metropolitana de Santa Fe y en la capital provincial generan un modelo de ciudad cada vez más difuso, caracterizado por el consumo creciente de recursos y la producción cada vez mayor de residuos y contaminación a los distintos elementos del sistema ambiental. Asimismo, el modelo de movilidad de personas, bienes y servicios imperante es el automotriz, medio de transporte que mayor cantidad de contaminación atmosférica genera en el entorno cercano y que más recursos renovables demanda (Cardozo, 2011). Otro de los factores que mayor impacto genera es el cambio en los usos del suelo. Espacios tradicionalmente rurales que se transforman en urbanos, que pasan a desempeñar funciones como la residencial de clase alta, la de servicios especializados e incluso industrial. Se inaugura, con estos procesos, un nuevo tipo de espacio donde se da el conflicto y la mezcla entre la morfología, estructura y funcionamiento de lo urbano con lo rural, lo cual constituye un verdadero punto de riesgo y alberga a una población en situación de vulnerabilidad creciente.

Finalmente, cabe remarcar que las problemáticas aludidas requieren de una decisión política que pasa tanto por el Estado, la academia y la sociedad civil, con relación a consensuar un estilo de desarrollo. Herrera et al.(1994), en su bello artículo "Las nuevas tecnologías y el futuro de América Latina. Riesgo y oportunidad", habla de la Buena Sociedad, caracterizándola como:

- igualitaria (en el acceso a bienes y servicios);

- participativa (todos los miembros tienen el derecho de tomar parte en las decisiones sociales en todos los niveles);

- autónoma (capaz de tomar decisiones basadas en sus propias aspiraciones y posibilidades);

- sin trabajo rutinario enajenante (las nuevas tecnologías permiten terminar esta forma de trabajo; el objetivo final será terminar con la presente división social compulsiva del trabajo) y;

- compatible con el medio ambiente físico (la compatibilidad deberá basarse no en medidas correctivas tomadas a posteriori, sino en la misma naturaleza del estilo de desarrollo).

Los usos de las nuevas tecnologías en la cuestión ciudadana y su relación con la extensión universitaria: desafíos y debates pendientes El tipo societal actual tiene como principio organizacional a la comunicación. En esta sociedad mediático-tecnológica, el ciberespacio aporta a interpretar nuestro posicionamiento en el mundo y, en simultáneo, condiciona la experiencia social. En ocasiones, las nuevas formas de comprensión mediadas por las tecnologías interactúan con diversos sujetos, lo que permitiría confirmar el crecimiento de la esfera pública y de nuevas formas de concebir lo político (Huergo, 2008). Hay quienes sostienen que las Ilamadas nuevas tecnologías permiten concebir y concretar acciones para el cambio social, dándose así un corrimiento desde la realidad digital hasta la realidad física. Otros afirman que lo que se produce es una falsa sensación de pertenencia y autonomía, ya que en ocasiones se establecen relaciones basadas únicamente en interacciones digitales.

La telefonía móvil y el ciberespacio, en sus múltiples manifestaciones, han posibilitado visibilizar conflictos, demandas y voces invisibilizadas, complejizando de esta manera los modos de entender la realidad. Incluso, a veces, son los mismos usuarios quienes dan a conocer sus versiones, inquietudes, motivaciones e
6) Declaraciones del director ejecutivo de Hábitat y Desarrollo, a raíz de la propuesta del gobernador electo por la provincia de Santa Fe, Miguel Lifschitz, acerca de la jerarquización gubernamental del área ambiental como Secretaría de Estado. Para más información, ver: www.ellitoral.com/ index.php/diarios/2015/10/31/opinion/ OPIN-02.html 
intereses personales sobre determinados aspectos y situaciones, dando cuenta así el ciberespacio de una polifonía de voces frente a los discursos dominantes.

A través de las cadenas de correo electrónico, de mensajes de texto, Twitter y WhatsApp, sus usuarios convocan a manifestaciones y marchas o solicitan firmas para peticionar por diferentes causas (por ejemplo, a través de la plataforma Change.org). Estas formas de lo político que constituyen parte de nuestras prácticas culturales han producido relatos y acciones que han tenido sus manifestaciones en el espacio público (cacerolazos, marchas que exigen justicia, movilizaciones en contra de la violencia de género —“Ni una menos"-, entre otras expresiones sociopolíticas).

Huergo acierta cuando afirma que:

"la cuestión no se define lisa y llanamente por el uso de las tecnologías, sino por las articulaciones que éstas poseen con nuevas modalidades de 'hacer política transformadora', con nuevas formas de comprensión del mundo y con nuevas maneras de la autonomía subjetiva". (2008:s/n ).

En cuanto a las prácticas de extensión universitaria, entre los actores que participan de las mismas, se encuentran las organizaciones de la sociedad civil. Las tecnologías de la comunicación se presentan a las OSC como herramientas de visibilidad, participación y difusión de sus acciones. Al pensar a estas organizaciones desde una mirada comunicacional, se las comprende como espacios en los que se fundan conversaciones.

"Estas conversaciones promueven intercambios para la creación de acciones, relaciones, interacciones y saberes colectivos. Pero para que resulte de este modo se requiere asentar el consenso, capaz de generar valores colectivos que, asumidos por todos o la mayoría, impregna el hacer organizacional de modo creativo y participativo". (Vargas y Zapata, 2010:81).

Ambas autoras se refieren a la comunicación comunitaria, cuyos medios de difusión son utilizados por los miembros de determinada comunidad, para cualquiera de los objetivos que éstos consideren pertinentes; sean fines de información, educación o esparcimiento. En ellos participan los habitantes como planificadores, productores o intérpretes. Son el instrumento de expresión de la comunidad, más que para la comunidad. La comunicación comunitaria procura designar un intercambio de puntos de vista y de noticias, y no una transmisión de una fuente a otra.

La extensión universitaria —desde sus múltiples dimensioneses una función que promueve y habilita el diálogo con la sociedad civil, el sector productivo y el Estado, para intentar contribuir a mejorar y ampliar la calidad de vida de los habitantes de determinado territorio.

En esta sociedad mediático-tecnológica, que tiene como principio organizacional a la comunicación, resulta imperioso que la extensión universitaria diseñe, implemente y observe (desde un compromiso ético y político) propuestas y recomendaciones de educación/ comunicación, atendiendo a la relación existente entre los actores sociales y las tecnologías de comunicación, en tanto herramientas de participación ciudadana y constructora-vehiculizadora de conocimientos. Dichas acciones invitan a la comunidad académica a pensar nuevos modos de construcción y apropiación social del conocimiento.

\section{Aporte de la Teoría Social}

Se trata de vincular, por un lado, a las múltiples perspectivas disciplinares, preocupadas en generar conocimientos desde una mirada comprensiva, intentando develar las causas y condiciones que producen la emergencia de las problemáticas, que desde la extensión se abordan. Por otro lado, como sostiene Larry Andrade:

"La realidad, nos interpela en tanto poseedores de un saber sobre lo social, pero esta interpelación encuentra sustento en la idea de que somos sensibles frente a lo social, a su movimiento. Siendo conscientes, a la vez que trabajar en torno a los problemas que atraviesan las sociedades contemporáneas, implica demarcarlas en relación a un campo de significados (como intereses, en torno a determinados fenómenos) que orientan nuestra mirada y a la vez pone en juego el compromiso que se asume frente a la urgencia de respuestas para problemas acuciantes. Captar las situaciones desde una concepción de totalidad, exige un esfuerzo que atraviese lo aparente y logre articular abstractamente aquello que le da existencia y especificidad". (2006:267)

\section{Perspectiva epistemológica}

Remite a los modos particulares en que se concibe la producción social de conocimientos, poniendo en diálogo el saber científicotecnológico con el mundo de vida de los actores sociales. Poner en diálogo la investigación y la intervención, posibilita la construcción de conocimientos, que puedan ser convalidados socialmente, mediante mecanismos que garanticen la participación activa de los actores, lo que a su vez requiere ser cuidadosos y respetuosos, desde la academia, en las decisiones metodológicas. Entendiendo que lo metodológico va más allá de lo instrumental, alude también a perspectivas tanto teóricas como epistemológicas y éticas. La intervención es permanentemente enriquecida con el aporte de la teoría social, las representaciones y los significados que para los actores implican las prácticas que despliegan. El ámbito de la práctica, de la acción, es el mundo de la vida y la realidad cotidiana, en un marco de relaciones intersubjetivas. Especialmente, interesa atender a la construcción social que los actores realizan del territorio y a las formas en que estos hacen uso de las instituciones en las que participan. $Y$ atender a las diferentes formas de apropiarse e intervenir el territorio de los actores e instituciones con los cuales, desde la extensión, se generan asociaciones, implica un modo particular de comprender el poder transformador 


\section{G6}

Poner en diálogo la investigación y la intervención, posibilita la construcción de conocimientos, que puedan ser convalidados socialmente, mediante mecanismos que garanticen la participación activa de los actores

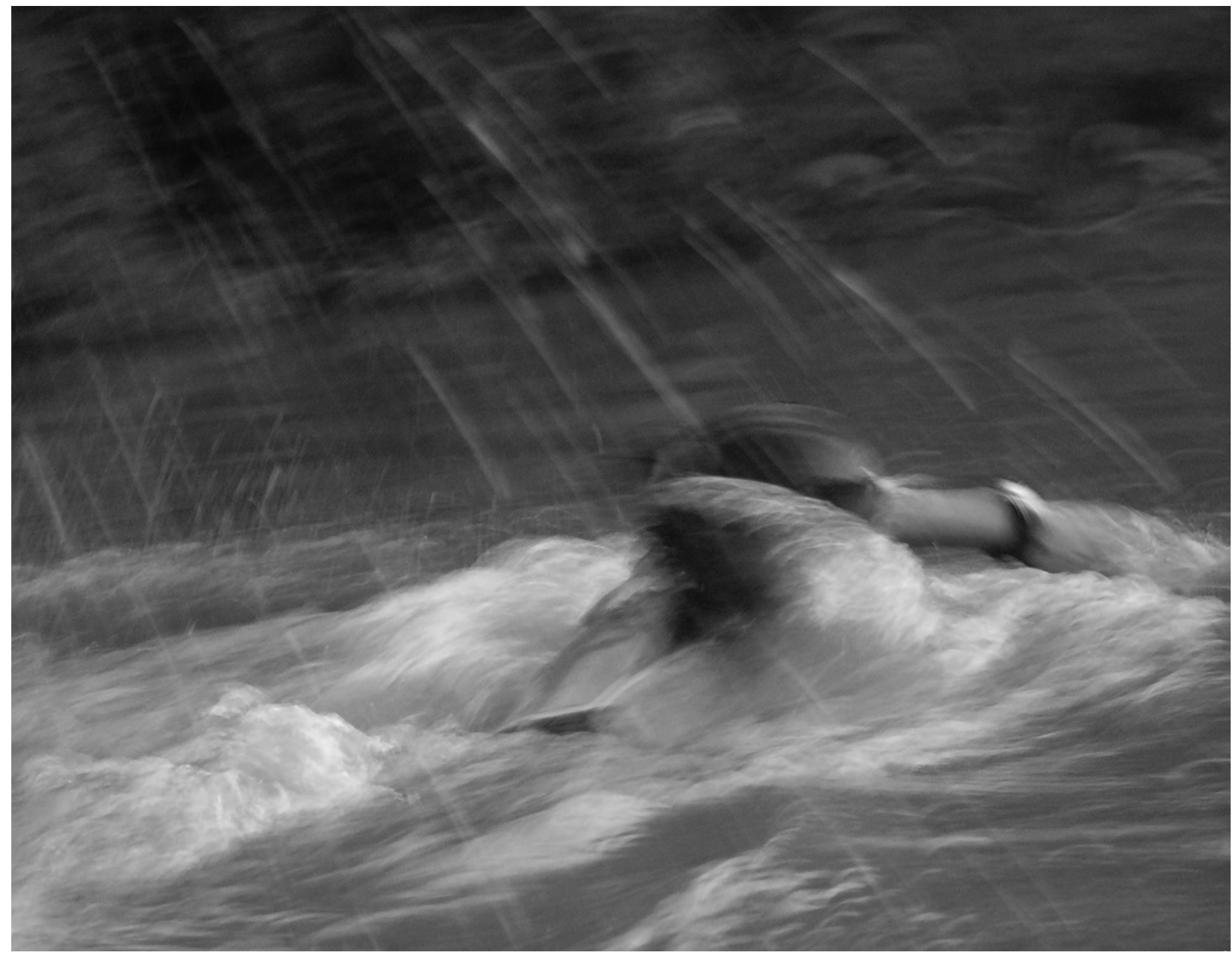

(c) Laura Malachesky 
de las prácticas de los agentes sociales. "Se trata de aprehender, esa realidad oculta, que solo se revela, encubriéndose, que no se deja ver sino bajo la forma anecdótica de las interacciones, en las cuales se disimula" (Bourdieu y Wacquant; 1995:23 y 24).

\section{Perspectiva ética}

Ligada al ejercicio de la ciudadanía y el fortalecimiento de las instituciones democráticas, como estrategia en la construcción de una sociedad de las singularidades, que implica, como sostiene Rosanvallon, garantizar a los individuos los medios de su singularidad, lo que conduce a su vez a replantear las políticas sociales:

"Más allá de las transferencias monetarias pasivas, que tienen una dimensión compensadora de la pérdida, de la insuficiencia o de la ausencia de un ingreso (subsidios por desempleo, ingresos de inserción, etc.) resulta prioritario equipar a los individuos, darles los medios de la autonomía. Implica, entonces, percibir las políticas sociales como dispositivos de constitución de sujetos". (2012:325)

Por otro lado, la crisis del presente no remite a una crisis de valores, como indica Castoriadis (1997) sino a una crisis del proceso identificatorio, ya que la creación de un sí mismo individual-social pasaba por lugares que fueron mutando y no existe (o no emerge) ninguna totalidad de significaciones imaginarias que pueda hacerse cargo de esta crisis de los apuntalamientos particulares. No hay una representación de la sociedad como morada de sentido y valor y como inserta en una historia pasada y futura. Trabajar por la inclusión y la cohesión social son un imperativo frente a un contexto donde las desigualdades se han profundizado; la brecha entre quienes más tienen y los que están en situaciones de vulnerabilidad se ha ampliado significativamente. Esto además se vincula con la debilidad de las democracias del presente, que no logran generar dispositivos para garantizar mayores niveles de inclusión social. El individualismo moderno desafía todas las formas colectivas de encuadramiento y el modo de articulación del individuo y lo colectivo que, sin sacralizar, conservó el "compromiso social" hasta principios de la década de 1970. Hoy asistimos al desarrollo de nuevos procesos de individualización con efectos contrastantes: por una parte, el individualismo positivo (autonomía, libertad, calidad de vida, felicidad), por otra, el desarrollo de un individualismo de masas socavado por la inseguridad y la falta de protecciones. Se trata de una paradoja cuya profundidad hay que sondear; uno vive más cómodo en su propia individualidad cuando ella está apuntalada por recursos objetivos y protecciones colectivas (Castel, 2010).

\section{Aspecto instrumental-operativo}

Si bien refiere a una cuestión metodológica, también hace a decisiones teóricas y éticas; implica una selección minuciosa de las técnicas e instrumentos que contribuyan a la identificación de las verdaderas causas del malestar en los escenarios de intervención.
Como de promover también mecanismos de participación, apropiación de conocimientos y aprendizajes, de garantizar procesos de convalidación social. Tiene que ver, a la vez, con generar espacios de socialización de información que aporten al capital social de las instituciones y territorios, en los que se despliegan las prácticas y se fundan trayectorias, en el campo social, donde se disputan espacios de poder.

\section{Reflexiones finales}

El presente trabajo intenta dar respuesta a un conjunto de interrogantes que sus autores se plantearon a partir de sus recorridos en el campo de la extensión y supone un esfuerzo por formular un modelo de intervención (a modo de marco referencial) pensado desde algunas claves conceptuales, epistemológicas y metodológicas, que le otorgan sentido.

La extensión universitaria, como una de las tres funciones sustantivas de la universidad, es objeto de análisis y debates, tanto en sus definiciones conceptuales, en su función social, como en las estrategias de intervención en escenarios atravesados por problemáticas sociales complejas que requieren de la construcción de perspectivas que aporten a su abordaje, con criterios de transformación y sustentabilidad.

Con tales premisas, los interrogantes planteados son los que mencionamos a continuación:

¿Cuáles son los determinantes conceptuales que definen la extensión universitaria? ¿Qué papel desempeña la teoría en los modos de concebir la extensión? ¿Cómo se teje el vínculo con el contexto por donde transita la cuestión social sobre el cual la propia acción extensionista se ve interpelada? ¿Qué papel asumen los equipos académicos y cómo se configura su rol como actor social dentro del propio espacio de intervención? ¿Qué incidencias tienen las intervenciones sociales en las problemáticas sociales que les dan origen? ¿En qué medida estas intervenciones se nutren de diagnósticos (cualitativos y cuantitativos) de las demandas sociales que el territorio anida? ¿Cuál es el sentido político actual de pensar la universidad en clave territorial y a nivel local?

Esto nos motivó a diseñar el modelo propuesto que, sin duda, podrá ser objeto de críticas como de aportes por quienes desarrollen acciones de extensión e igualmente podrá generar nuevos interrogantes a modo de desafíos o, en palabras de Teresa Matus: "La apuesta es la construcción de una lógica de innovación en los procesos de intervención social, que profundice en nuevos modelos complejos de intervención, que evalúe sus estrategias, su consistencia operacional, sus mecanismos para una gestión más integral que se oriente a resultados. Que se adentre en preguntas nuevas y tenga como resultado una intervención más competente y sólida, que se inserte en una perspectiva de los derechos, promueva una participación responsable y fomente la autonomía de los sujetos". (2012:58) 


\section{Referencias bibliográficas}

Ackerman, M. (2007). El trabajo, los trabajadores y el derecho del trabajo. Recuperado de: http://www.trabajo.gov.ar/left/estadisticas/descargas/revistaDeTrabajo/2007n04_revistaDeTrabajo/2007n04_a04_meAckerman.pdf (consultado el 26 de agosto de 2016).

Alonso, G. y Di Costa, V. (2011). Cambios y continuidades en la política social argentina 2003-2010. Ponencia preparada para su presentación en el VI Congreso Argentino de Administración Pública, Resistencia, Chaco, 6, 7 y 8 de julio.

Andrade, L. (Comp.) (2006). Lo social inquieto (e inquietante) objeto. Aportes para pensar. Buenos Aires: Miño y Dávila Editores.

Andrenacci, L. y Soldano, D. (2006). Aproximaciones a la Teoría de la Política Social a partir del caso argentino. En Andrenacci, L. (Comp.), Problemas de política social en la Argentina contemporánea. Buenos Aires: Prometeo, UNGS.

Barnes, B. and Edge (1982). Science in Context: readings in sociology of science. The Open University Press, Milton Keynes.

Bloor, D. (1976). Knowledge and social imaginery. London: Routledge.

Bourdieu P.; Wacquant J. D. (1995). Respuestas. Por una Antropología Reflexiva. Buenos Aires: Grijalbo.

Callon, M. y Latour, B. (1986). Les paradoxes de la modernité. Comment concevoir les innovations? Prospective et Santé, (36), 13-25.

Carballeda, A. (2002). La intervención en lo social. Exclusión e integración en los nuevos escenarios sociales. Buenos Aires: Paidós.

- (2005). La intervención en lo social. Buenos Aires: Espacio.

Cardozo, M. M. (2011). Impacto ambiental de los nuevos procesos urbanos en el área metropolitana de Santa Fe. Ponencia presentada en el marco V Congreso Iberoamericano sobre Desarrollo y Ambiente de REDIBEC, $V$ Jornadas de la Asociación Argentina Uruguaya de Economía Ecológica del Congreso CISDA. Facultad de Ingeniería y Ciencias Hídricas. Universidad Nacional del Litoral. Santa Fe, Argentina

Castel, R. (2010). El Ascenso de Las Incertidumbres: Trabajo, Protecciones, Estatuto del Individuo. Buenos Aires: Fondo de Cultura Económica.

Castoriadis, C. (1997). El avance de la insignificancia. Buenos Aires: Eudeba. CELS (2017). Derechos Humanos en la Argentina. Informe 2017. Buenos Aires: Siglo XXI Editores.

Collins, H. M. (1983). The sociology of scientific knowledge: studies of contemporary science,en Annual Review of sociology, 9, 265-285. Recuperado de: http:// www.annualreviews.org/doi/abs/10.1146/annurev.so.09.080183.001405 (consultado el 20 de marzo de 2017).

Danani, C. y Hintze, S. (Coords.) (2013) Protecciones y desprotecciones II: Problemas y debates de la seguridad social en Argentina. Buenos Aires: UNGS.

Grassi, E. (2000). Procesos político-culturales en torno al trabajo. Acerca de la problematización de la cuestión social en la década del 90 y el sentido de las solucionespropuestas. Sociedad, (16). Buenos Aires: Facultad de Ciencias Sociales, UBA. Harvey, D. (2004). El nuevo imperialismo: Acumulación por desposesión. Socialist Register, 40, 99-129.
Herrera, A. y otros (1994). Las nuevas tecnologías y el futuro de América Latina. Riesgo y oportunidad. Buenos Aires: Siglo XXI Editores.

Huergo, J. (2008). Culturas mediático-tecnológicas y campo formativo. Recuperado de: http://www.margen.org/suscri/margen51/huergo.html (consultado el 17 de agosto de 2016).

Isuani, A. (2015). Capitalismo Vs. Democracia. El caso argentino. Buenos Aires: Eudeba.

Matus, T. (2012). Apuntes sobre intervención social. Recuperado de: https://trabajosocialucen.files.wordpress.com/2012/.../apuntessobreintervencionsocial Mazzuca, S. (2012). Recursos naturales, populismo rentista y tentaciones hegemónicas en América del Sur. Araucaria, (29).

Menéndez, G. (2011). Los desafíos presentes y futuros de la extensión universitaria. Revista $+E,(1), 24$.

- (2012). Extensión y políticas públicas: la universidad en el centro del debate. Revista $+E,(2), 9$.

Neffa, J. C.; Oliveri, M.; Persia, J. (2010). Transformaciones del mercado de trabajo en Argentina: 1974-2009. Atlántida: revista canaria de Ciencias Sociales, 10(2), 19-48. Recuperado de: http://publica.webs.ull.es/upload/REV\%20ATLANTIDA/2\%20\%202010/01\%20Julio\%20Cesar\%20Neffa\%20y\%20otras.pdf http://www.iirsa.org/Page/Detail?menultemld=121http://www.iirsa.org/Page/Detail?menultemld=121

UNL. Secretaría de Extensión de la Universidad Nacional del Litoral (s/f). Sistema integrado de Programas y Proyectos de Extensión. Recuperado de: http://www. unl.edu.ar/extension/wp-content/uploads/sites/4/2016/11/293_sistema-integrado-de-programas-y-pro.pdf (consultado el 9 de abril de 2017).

Repetto, F. (2008). Política social y redistribución en la Argentina: la historia reciente, los retos futuros. Buenos Aires: Mimeo.

(2014). Políticas sociales: una mirada político-institucional a sus reformas, desafíos e impactos. En Acuña, C. H. (Comp.), El Estado en acción. Fortalezas y debilidades de las politicas sociales en la Argentina. Buenos Aires: Siglo XXI Editores. Rofman, A. (2016) (Comp.). Participación, políticas públicas y territorio. Aportes para una perspectiva integral. Buenos Aires: UNGS.

Rosanvallon, P. (2012). Una sociedad de iguales. Buenos Aires: Manantial. Schavelzon, S. (2015). Plurinacionalidad y Vivir Bien/Buen vivir. Dos conceptos leídos desde Bolivia y Ecuador post-constituyentes. Quito: Abya-Yala.

Subirats, J.; Knopfel, P.; Larrue, C.; Varonne, F. (2008). Análisis y gestión de las políticas públicas. Barcelona: Ariel.

Svampa, M. (s/f). La disputa por el desarrollo: territorio, movimientos de carácter socio-ambiental y discursos dominantes. Recuperado de: http://www.maristellasvampa.net/archivos/ensayo43.pdf (consultado el 5 de mayo de 2017).

Vargas, T. y Zapata, N. (2010). Enredando prácticas. Comunicación desde las organizaciones sociales. Buenos Aires: San Pablo.

Zelanik, J. (2012). Las coaliciones kirchneristas. En Malamud, A. y De Luca, M., La politica en tiempos de los Kirchner. Buenos Aires: Eudeba. 